
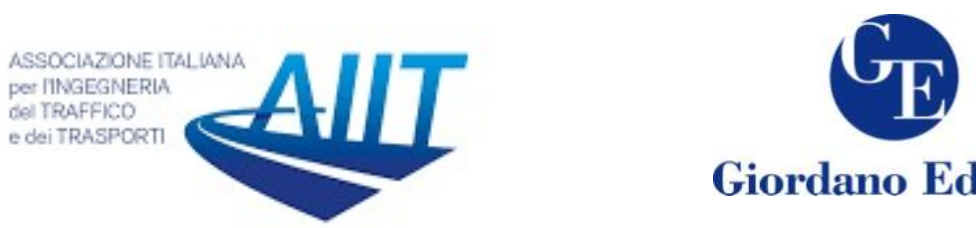

Giordano Editore

\title{
Physiological and driving behaviour changes associated to different road intersections
}

\author{
Natalia Distefano ${ }^{1 *}$, Salvatore Leonardi ${ }^{1 *}$, Giulia Pulvirenti ${ }^{1 *}$, \\ Richard Romano ${ }^{2}$, Natasha Merat ${ }^{2}$, Erwin Boer ${ }^{2}$, Ellie Woolridge ${ }^{3}$ \\ ${ }^{\prime}$ Department of Civil Engineering and Architectural, University of Catania, Via Santa Sofia 64, Catania, \\ 95125, Italy \\ ${ }^{2}$ Institute for Transport Studies, University of Leeds, Leeds, United Kingdom \\ ${ }^{3}$ Transport Systems Catapult, Milton Keynes, United Kingdom
}

\begin{abstract}
Road traffic injuries claim more than 1.2 million lives each year in the world and have a huge impact on health and development. It is commonly acknowledged that the human factor and the interaction between the human factor and the road environment are among the most common causes of road accidents. Intersections are among the most complex road environments: their geometric and traffic characteristics weigh the driver workload, affecting the driving behaviour and consequently the risk of accident. This study intends therefore to contribute for a better understanding of the relationship between different types of intersection and the human factor. The ultimate aim is to understand how at grade intersections affect the driving behaviour by comparing the drivers' stress level for roundabouts and standard intersections. Electrodermal activity can provide a real-time assessment of the driver's stress level. Electrodermal activity was therefore collected continuously during a driving study which took place on a test environment based at Cranfield University and surrounding roads. Twenty participants were involved within the study. The analysis focused on four crossing manoeuvres on three at grade intersections (two $\mathrm{T}$-junctions and a roundabout) situated on the study location. Results showed that the number of SCR peaks as well as the amplitude of the peaks are overall higher for the two manoeuvres on the roundabout. The stress level induced by each type of intersection was evaluated through an Electrodermal Impact Index which takes into account both the number and the amplitude of SCR peaks. Results suggested that the stress level induced by roundabouts is more than double that induced by standard intersections.
\end{abstract}

Keywords: road safety; electrodermal activity; skin conductance; roundabouts; T-junctions; human factor; drivers' stress level.

\section{Introduction and background}

Hazard perception, i.e. the ability to "read the road" in relation to potentially dangerous situations in the traffic environment, seems to be the only component of

\footnotetext{
* Corresponding author: Salvatore Leonardi (s.leonardi@unict.it)
} 
driving skills that has been found to be related to accident involvement (Horswill and McKenna, 2004). For many decades road safety researchers have been attempting to explain how people perceive and understand risk (McKenna and Crick, 1997; Borowsky et al., 2010). The reason that hazard-perception ability has retained interest over the years is because anticipation of hazardous traffic situations is perhaps one of the major contributions to driver safety. It is commonly acknowledged that human factors may contribute to accident involvement in traffic (Grayson and Maycock, 1988). Based on a study of 2041 traffic accidents, Sabey and Taylor (1980) concluded that human factors were contributing elements in $95 \%$ of the accidents. Driving behaviour was identified as the most central of these factors. Driving attitude, which is manifested by driving behaviour, strongly affect the hazard perception of drivers (Cheng et al., 2011). It is therefore important to understand the various aspects that affect the drivers' ability to perceive danger and risk and, thus, that affect their driving behaviour. Human driving behaviour, and so hazard perception ability, is strongly conditioned by the road environment in its entirety, as driving involves complex interactions between the driver and the environment. Considering the extreme complexity of road networks, the aspects influencing the driving behaviour should be studied with specific regard to the elements of road networks as, for example, intersections. The meeting point of two or more roads is the focus of conflict since the beginnings of regular traffic. Intersections are among the most complex road environments: their geometric configuration, the signs and markings, the road furniture, the qualitative and quantitative characteristics of traffic, the vehicular conflicts are all elements which weigh the driver workload, conditioning the driving behaviour and, consequently, affecting the risk of accident (Distefano and Leonardi, 2018). The safety benefit of roundabout conversions has been recognized world-wide. Several studies to date have examined the safety implications of replacing standard intersections with roundabouts. Converting junctions to roundabouts has been found to reduce the number of accidents, in particular fatal accidents (Elvik, 2003; Persaud et al., 2001; Vujanić, 2016). Studies of roundabouts in various countries have shown that roundabouts can significantly improve functional characteristics (Easa and Mehmood, 2006; Ma et al., 2013), as well as traffic safety (Chen et al., 2013; Gross et al., 2013). Lot of researchers also examined the public opinion on roundabouts (Distefano et al, 2019a; Distefano et al., 2019b; Hu et al., 2014; Retting et al., 2002). and the relationship between geometric elements and safety benefits in roundabouts (e.g. Daniels et al., 2010, Distefano et al., 2018). While previous studies investigated the benefits of converting junctions into roundabouts as for crash rates and traffic conditions, to the best of the authors' knowledge, no studies analysed how drivers' stress level change between standard intersections and roundabouts. Research on user behaviour and perceptions can be a helpful tool in improving road safety and accident prevention. The aim of this study is to evaluate drivers' physiological and behavioural responses in order to assess drivers' risk perception for standard intersections and roundabouts and compare them. The authors indeed believe that there is a need to deepen the human interactions with the road environment for different intersections. This paper presents a method for measuring and quantifying drivers' overall stress in a real environment using physiological signals. Physiological signals are a useful metric for providing feedback about a driver's state because they can be collected continuously and without interfering with the driver's task performance and with the drivers' perception of the road. When humans are subjected to stressors, they tend to show a variety of physiological responses such as pupil dilation, increased heart rate, slowed 
digestion, and a constriction of blood vessels, mechanisms that are collectively known as the 'fight-or-flight' response (Cain, 2007; Wickens et al., 2004). Measuring drivers' physiological responses during periods of effort investment in simulators and real vehicles has been a subject of investigations for several decades. Examples include physiological measurements during the presence/absence of a secondary task (Mehler et al., 2009), as a function of road infrastructure (Dijksterhuis et al., 2011), or for different levels of automated driving (De Winter et al., 2014). The Autonomic Nervous System (ANS), responsible for involuntary activities, is made up of Sympathetic and Parasympathetic nervous systems. Stressful events or emergency situations cause dynamic changes in ANS, where the activity rate in the Sympathetic Nervous System (SNS) increases and the Parasympathetic Nervous System (PNS) activity decreases. Alternatively, activities in the PNS dominate during resting activities. SNS and PNS regulate the electrodermal activity, heart rate variability, and brain waves, which are the main measures for stress used in literature, and other physiological systems including blood pressure. Electrodermal activity, also known as galvanic skin response or skin conductance, is a reliable indicator of stress (Seyle, 1956). Electrodermal activity (EDA) refers to the variation of the electrical properties of the skin in response to sweat secretion. The whole mechanism is controlled by the sympathetic nervous system and can be used to control the functionality of the cognitive system. By applying a low constant voltage, the change in skin conductance (SC) can be measured non-invasively (Fowles et al.,1981). The time series of SC can be categorized into two components: tonic (i.e., skin conductance level; SCL) and phasic components (i.e., skin conductance response SCR) that have different time scales and relationships to external stimuli (Cacioppo et al., 2007). Specifically, SCR is a useful index of an individual's perceived risk. SCR could be a useful indicative of activities of the sympathetic branch of the autonomic nervous system because the sweat glands are innervated by the sympathetic nervous activities (Poh et al., 2010). The sympathetic arousal stimulated by external stressors could be reflected by a higher SCR. In this sense, EDA has been used to understand an individual's mental status related to sympathetic arousal (e.g., stress, attention, risk perception, etc.) in various situations such as occupational setting, human-computer interaction, traffic and automation, and marketing and product evaluation (Boucsein, 2012). SCR could be a more useful index of the perceived risk than other physiological signals such as heart rate, respiration rate, and skin temperature because SCR is the only autonomic physiological variable that is not contaminated by the parasympathetic branch of the autonomic nervous system (Braithwaite et al., 2013).

\section{Method}

\subsection{Participants}

Twenty-three staff members were recruited from Cranfield University, three individuals participated within pilot trials and twenty individuals participated within the trials. An advert was placed on the Cranfield University website, and participants who showed interest were sent an email which included information about the study and a participant recruitment questionnaire. The questionnaire data was used to determine the appropriate participant sample and participants were invited to participate within the study. The twenty participants involved within the trial were evenly divided between males and females. Participants were aged twenty-eight to fifty years of age. They were required to have held a UK driving licence for a minimum of three years. One 
participant was excluded from the analysis because of a problem during the data collection. The final sample therefore consisted of nineteen participants (ten males and nine females). An ethics application was made for the experiment to the Research Ethics committee at Cranfield University and received approval. Participants gave their informed consent to take part in the experiment. They were informed that all information collected would have been dealt with in the strictest confidence and would have only been used for research purposed. Participants were also informed that they would have not been judged as for their ability as drivers and that the only aim of the study was to analyse the behaviour of a group of drivers to draw conclusions about drivers in general.

\subsection{Experiment design}

The trial was a naturalistic user study which aimed to explore and capture the user's natural behaviours in the real-world. The experiment was part of the "HumanDrive" project. The "HumanDrive" project goal is to develop driverless vehicle technologies that can deal with varied UK driving scenarios in a more humanlike way. The ego vehicle driven by the participant was a Nissan Leaf. The vehicle was instrumented with an OXTS RT1003 vehicle localisation system (which allowed to record vehicle position, forward speed, linear acceleration and GPS time at $100 \mathrm{~Hz}$ ) and three colour cameras (one forward facing, one driver facing, one steering wheel facing). An Empatica E4 wrist band sensor was worn by the participant to collect physiological data which records their blood pulse volume and their skin conductance. The study involved time for the participants to familiarise themselves with the vehicle, participant 'within trial' data collection, followed by interviews to further understand their driver behaviour. Before the drive could be carried out the participant had to be familiar with the vehicle and how to control it. The ego vehicle dimensions, operation and electric drive train may be new to the participant. Therefore, a tutorial was provided to explain how the vehicle works, whilst the vehicle was stationary. Moreover, a familiarisation period was built into the study to ensure that participants had adequate time to get familiar with the vehicle and a similar level of familiarisation was achieved across all participants to prevent experimental bias. The familiarisation drive was accompanied by a facilitator, sitting in the back of the vehicle behind the driving seat and issuing directions. The facilitator had to confirm during and/or at the end of the familiarisation drive that the participant was confident driving. After this phase the driving study started. Participants were asked to drive naturally. As well as the familiarisation drive, directions were issued by the facilitator who was sat directly behind the driver's seat. Trials took place between 9am and $4 \mathrm{pm}$, to ensure similar and bright visibility and avoid busy commuter traffic. When experience adverse weather such as heavy rain or snow were experienced, the trial was postponed.

\subsection{Study location}

The driving study took place on the MUEAVI (Multi-User Environment for Autonomous Vehicle Innovation) test environment based at Cranfield University and surrounding roads. MUEAVI is a controlled and instrumented stretch of road, located on the edge of the University Campus. Both public roads and campus roads link to the MUEAVI facility, these roads were also incorporated within the trial, particularly to further assess interactions with the roundabouts and intersections. Fig. 1 shows the study location and the driving route. It can be seen the driving route composed by the 
loop around Cranfield and the MUEAVI (central quadrant) and the familiarisation drive (upper left quadrant). Participants drove the route different times continuously and therefore they made different manoeuvres on the different intersections situated on the driving route. The present study focuses on the drivers' risk perception of three intersections situated on the study location. These intersections are the roundabout $\mathrm{R}$ and the two $\mathrm{T}$-junctions $\mathrm{T} 1$ and $\mathrm{T} 2$ shown in Fig. 1. The roundabout has three perpendicular legs and a diameter of 45 meters approximately. The T-junctions have the three legs perpendicular to each other and have similar dimensions. The analysis made regards the drivers' stress level during a crossing manoeuvre for each T-junction (manoeuvre 1 and manoeuvre 2) and during two crossings manoeuvres on the roundabout (manoeuvre 3 and manoeuvre 4). We chose to compare crossing manoeuvres, rather than turning right or turning left manoeuvres, because the speed for crossing manoeuvres is higher than for other manoeuvres. Since the final aim of the study was to evaluate how the type of intersection affects drivers' risk perception, we analysed only the manoeuvres where the traffic was not affecting the driving behaviour (no traffic or really low traffic at the intersection during the execution of the manoeuvres).

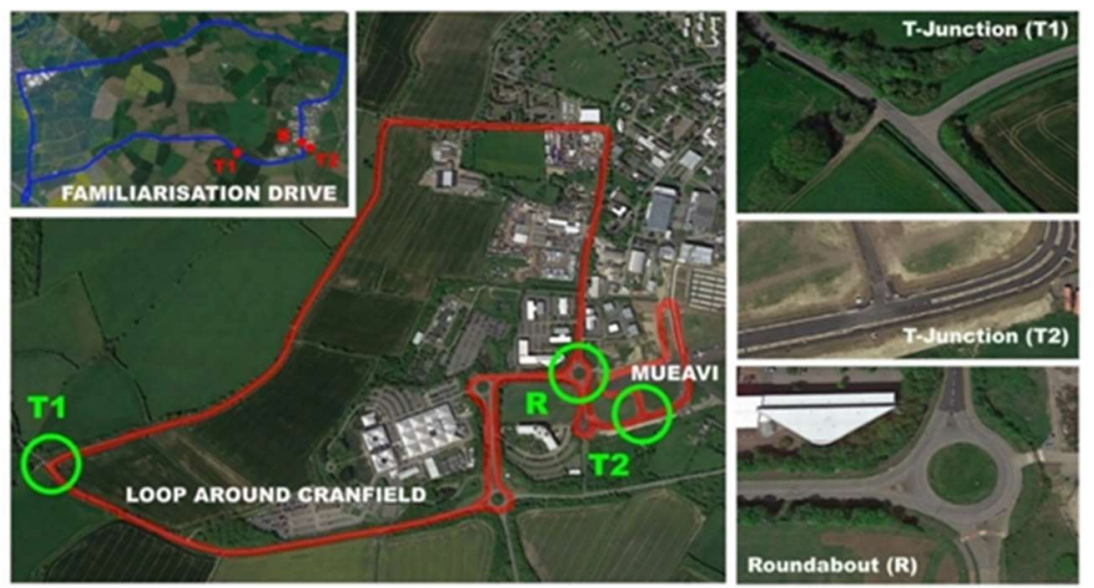

Figure 1: Study location.

\subsection{EDA recording}

Recent advancements in wearable technologies allowed to overcome the limitations of traditional EDA sensors. Wearable sensors (e.g., off-the-shelf wristband-type wearable sensor) can continuously collect drivers' physiological signals without affecting driving conditions. Empatica E4 wristband (Empatica Inc., Cambridge, MA, USA) was used to record EDA continuously and unobtrusively during the experiment. Participants wore the wristband on their right wrist. In contrast to lower-accuracy, consumer-oriented wristband sensors available on the market - typically for fitness tracking - the E4 is a research quality multi-sensor wristband (Garbarino et al., 2014). The wristband embeds four sensors: EDA, photo-plethysmograph, thermometer, and accelerometer. The E4 wristband EDA sensor uses the exosomatic method, which measures skin conductance in microSiemens $(\mu \mathrm{S})$ by applying a small external current. The sampling frequency of the EDA sensor is $4 \mathrm{~Hz}$ (i.e., four samples per second). 


\subsection{Data analysis}

As aforementioned, EDA can be decomposed by a slowly varying tonic activity (i.e., skin conductance level; SCL) and a fast-varying phasic activity (i.e., skin conductance response SCRs). The tonic level, known as skin conductance level (SCL), slowly varies and changes slightly on a time scale of tens of seconds to minutes. The rising and declining SCL is constantly changing within an individual respondent, depending on their hydration, skin dryness, or autonomic regulation. The tonic level can also differ markedly across individuals. This has led some researchers to conclude that the actual tonic level on its own is not that informative (Braithwaite et al., 2013). The phasic response rides on top of the tonic changes and shows significantly faster alterations. Variations in the phasic component are visible as EDA peaks. The phasic response is also labelled skin conductance response (SCR) as it is sensitive to specific emotionally arousing stimulus events (event-related SCRs, ER-SCRs). These bursts occur between 1-5 seconds after the onset of emotional stimuli. By contrast, non-specific skin conductance responses (NS-SCRs) happen spontaneously in the body at a rate of 1-3 per minute and are not a consequence of any eliciting stimulus. SCRs may reflect stimulusspecific responses or non-specific responses. An SCR shows a steep incline to the peak and a slow decline to the baseline. The succession of SCRs usually results in a superposition of subsequent SCRs, as more often than not, a subsequent SCR occurs during the decay of a previous one. Hence SC does not show distinct peaks of phasic activity, but rather is characterized by the superposition of extended responses, which eventually complicates the assessment of responses (Boucsein, 2012). Continuous decomposition analysis (Benedek and Kaernbach, 2010) was used in this study for extracting SCRs peaks, as it enables separate detection of superimposed responses. The data were therefore analysed by determining the number of peaks in the skin conductance response (SCR) that subjects had while driving on each intersection through the following procedure. The phasic data (SCR) was extracted from the EDA signal by using a median filter. For each sample, the median EDA score of the surrounding samples was calculated based on a $+/-4$ seconds interval centred on the current sample. This value was then subtracted from the current sample to obtain the phasic data. Peaks onset/offset thresholds were set to $0.01 \mu \mathrm{S}$ and $0 \mu \mathrm{S}$ respectively (Benedek and Kaernbach, 2010). Peak onset value represents the starting point in time where a peak is detected, while the offset value represents the time when a peak has passed. To avoid false positives, the onset value was not counted if it is less than 0.01 $\mu \mathrm{S}$. The maximum original EDA data within each pair of onsets and offsets is an SCR peak. SCR peak amplitude is the amplitude at the peak divided the amplitude at onset. A peak was only considered if its amplitude was higher than the threshold amplitude by 0.005 above the onset value. Also, a signal jump threshold that accounts for false peaks - caused by noise - is set to $0.02 \mu \mathrm{S}$. After continuous decomposition analysis, onsets for each individual SCR were obtained. Onsets served as the basis to subsequently calculate the number of SCR peaks and their amplitude, using a moving window approach with a temporal window corresponding to $100 \mathrm{~m}$ before and $100 \mathrm{~m}$ after each intersection. QGIS 3.6 was used in order to identify the temporal window corresponding to $100 \mathrm{~m}$ before and $100 \mathrm{~m}$ after each intersection starting from the GPS traces. 


\section{Results}

Fig. 2 shows the number of SCR peaks (Fig. 2 a) as well as the average amplitude of peaks (Fig. 2 b) of all subjects during each crossing manoeuvre on the two T-junctions (manoeuvres 1 and 2) and during each crossing manoeuvre on the roundabout (manoeuvres 3 and 4). For statistical analysis, initially descriptive statistics were calculated for the SCR through SPSS 24.0. For further SCR data treatment, Levene's test was performed. The Levene's test results for SCR measurements in terms of average peaks amplitude for the two groups (T-junctions and roundabouts) were: Levene statistic $=1.675 ; \mathrm{df1}=1.00 ; \mathrm{df} 2=85$; corresponding $\mathrm{p}$ value $=0.199(>0.05)$. According to the Levene's test, variances are equal for the two groups and the criterion of homogeneity is met. We proceeded with Kolmogorov-Smirnov testing for the normality of each group of data and the results showed that both data series were not parametric. For the purposes of comparison between groups, we used a Kruskal-Wallis test for the two groups and measured their SCR (peaks amplitude). The Kruskal-Wallis test results indicate that there is a statistical difference between groups with $\mathrm{p}$ value $=$ 0.047 (< 0.05). For further investigation we used a Mann-Whitney test. A statistical difference was found between the two groups (Mann-Whitney $U=620.00$; Wilcoxon $W$ $=1055.00 ; \mathrm{Z}=-1.990$; corresponding $\mathrm{p}$ value $=0.047<0.05)$. Finally, the Two-Sample Kolmogorov-Smirnov Test showed a statistical difference between the two data series (Most Extreme Differences: Absolute $=0.328$, Positive $=0.034$, Negative $=-0.328$; Kolmogorov-Smirnov $\mathrm{Z}=1.440$; corresponding $\mathrm{p}$ value $=0.032<0.05$ ). In order to evaluate the overall influence of roundabouts and T-junctions on drivers' risk perception, the total number of SCR peaks and the average amplitude of SCR peaks for the manoeuvres on the $\mathrm{T}$-junctions and for the manoeuvres on the roundabout were calculated for each participant (Fig. 3 a and Fig. 3 b). From the analysis of Fig. 2 and Fig. 3 it can be seen that thirteen out of nineteen participants had more SCR peaks during the manoeuvres on the roundabout rather than during the manoeuvres on the Tjunctions; ten of them also had a higher amplitude of the peaks during the manoeuvres on the roundabout. Eleven out of nineteen participants show a higher amplitude of the SCR peaks during the manoeuvres on the roundabout. Four participants had the same number of peaks during the manoeuvres on the roundabout and during the manoeuvres on the T-junctions. One participant (participant 5) did not show any peak at all. Only one participant had more SCR peaks during the manoeuvres on the T-junctions rather than during the manoeuvres on the roundabout (participant 11). The number of SCR peaks as well as the amplitude of the peaks are overall higher for the manoeuvres on the roundabout (number of peaks: 58 for roundabout, 29 for T-junctions; medium amplitude of peaks: 1.132 for the manoeuvres on the roundabout, 1.083 for the manoeuvres on the T-junctions. These results can be interpreted for increased anxiousness (higher emotional response) with respect to baseline (Villarejo et al. 2012), with more impact in the roundabouts. 

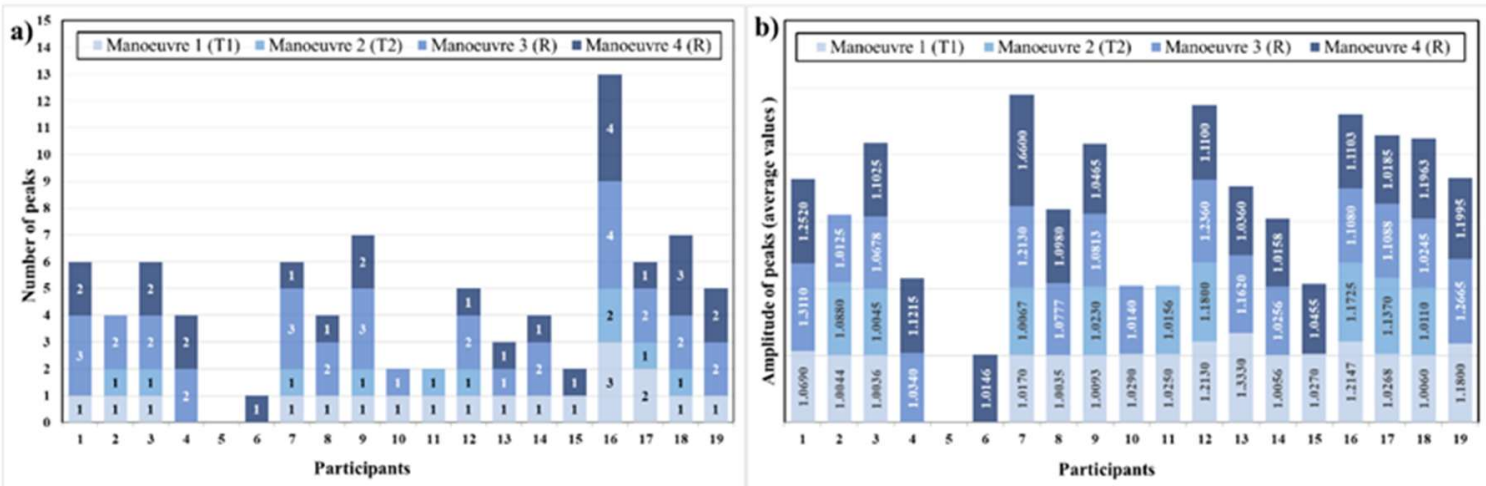

Figure 2: (a) Number of SCR peaks of all subjects during each crossing manoeuvre on the two T-junctions (T1 and T2) and during each crossing manoeuvre on the roundabout (R); (b) average amplitude of SCR peaks of all subjects during each crossing manoeuvre on the two T-junctions (T1 and T2) and during each crossing manoeuvre on the roundabout $(\mathrm{R})$.
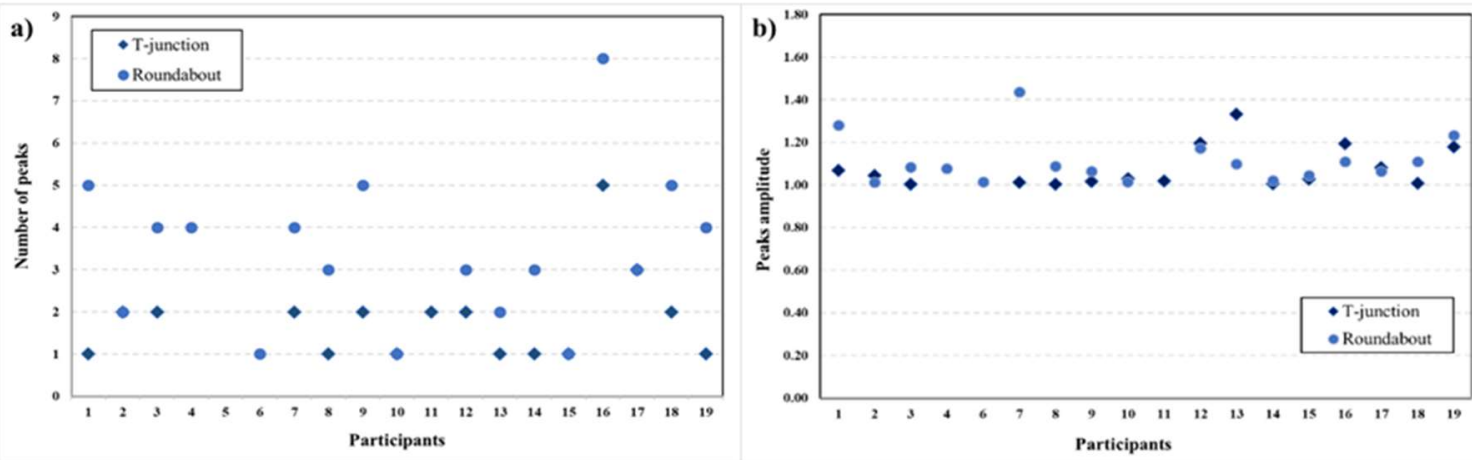

Figure 3: (a) Total number of SCR peaks of all subjects for the manoeuvres on the Tjunctions and for the manoeuvres on the roundabout; (b) average amplitude of SCR peaks of all subjects for the manoeuvres on the T-junctions and for the manoeuvres on the roundabout.

In order to further assess drivers' risk perception for T-junctions and roundabouts and to evaluate the stress level induced by each type of intersection, an index representing the driver's stress level at each type of intersection was defined. This index, named Electrodermal Impact Index, was calculated as the product of the number of SCR peaks and the average amplitude of SCR peaks (Table 1). We obtained an Electrodermal Impact Index of 65.66 for the crossing manoeuvres on the roundabout and an Electrodermal Impact Index of 31.41 for the crossing manoeuvres on the T-junctions. The ratio between these two Electrodermal Impact Index shows that roundabouts are affecting the drivers' stress level and so the drivers' risk perception 2.1 times more than T-junctions.

Table 1: Intersection Impact Factors for roundabouts and for T-junctions.

\begin{tabular}{cccc}
\hline Intersection Type & $\begin{array}{c}\text { Average amplitude of SCR } \\
\text { peaks }\end{array}$ & Number of SCR peaks & $\begin{array}{c}\text { Electrodermal Impact } \\
\text { Index }\end{array}$ \\
\hline Roundabout & 1.132 & 58 & 65.66 \\
\hline T-Junction & 1.083 & 29 & 31.41 \\
\hline
\end{tabular}




\section{Discussion and conclusion}

Roundabouts strategically modify the built environment to affect traffic speed and patterns. They can therefore be fully considered part of traffic calming measures as they influence the users' driving behaviour by inducing a certain level of stress. It is well acknowledged that roundabouts, similarly to other traffic calming measures (speed tables, chicanes, road narrowing, speed bumps, etc.), cause significant reductions of speeds, of accidents and of the severity of the accidents themselves (Fernandes et al., 2018, Distefano and Leonardi, 2019, Nicholson et al., 2014). Recent studies also confirmed that the effectiveness of traffic calming measures can also induce transport mode choices alternative to motor vehicles, favouring sustainable transport modes such as cycling and walking (Gössling et al., 2019, Distefano et al., 2019c, Vasudevan and Patel, 2017, Nicholson et al., 2014).

It is even more evident how roundabouts affect drivers' behaviour if a comparison is made between the accident rates of roundabouts and of standard intersections. The present study examined how at grade intersections affect the driving behaviour by comparing the drivers' stress level for roundabouts and standard intersections. The parameter used for estimating the human response to the stress coming from the two different types of intersection was the Electrodermal activity (EDA) measured on a sample of 19 drivers while driving two crossing manoeuvres on a roundabout and two crossing manoeuvres on two standard intersections. The finding showed that the number of SCR peaks as well as the amplitude of the peaks are overall higher for the two manoeuvres on the roundabout. The stress level induced by each type of intersection was evaluated through an Electrodermal Impact Index which takes into account both the number and the amplitude of SCR peaks. The results are particularly interesting as they suggested that the stress level induced by roundabouts is more than double that induced by standard intersections. Therefore, this research has enabled us to quantify the positive effects of roundabouts in terms of safety through an approach that allowed to evaluate different type of at grade intersections taking into account a parameter directly linked to the human factor. That is a confirmation of a lot of studies demonstrating that safety perception for roundabouts is higher than intersections at grade (Leonardi et al., 2019, Gross et al., 2013). Human factors, which are broadly recognized as the main cause in determining road accidents, can be further examined by means of other indicators of the drivers' stress level. Physiological signals are indeed a useful metric for providing feedback about a driver's state. In this paper the authors analysed only the Electrodermal activity of the drivers to evaluate their physiological and behavioural responses to different intersections. Further studies will deepen the correlations between different at grade intersections and other physiological parameters, such as blood volume pulse, heart rate and heart rate variability, which were measured during the same experiment on which this study is based.

\section{Acknowledgements}

The work described in this paper was undertaken in connection with the HumanDrive project which is co-funded by Innovate UK, the UK's innovation agency. This paper is published with kind permission from the HumanDrive consortium: Nissan, Hitachi, Horiba MIRA, Atkins Ltd, Aimsun Ltd, SBD Automotive, University of Leeds, 
Highways England, Cranfield University and the Transport Systems Catapult. Cranfield University performed the data collection for the experiment. Innovate UK do not approve the technical content of academic papers.

This work was also partially financed by the University of Catania within the project "Piano della Ricerca Dipartimentale 2016-2018" of the Department of Civil Engineering and Architecture.

\section{References}

Boucsein, W. (2012) "Electrodermal Activity", Springer Science \& Business Media, New York, NY.

Borowsky, A., Shinar, D., Oron-Gilad, T. (2010) "Age, skill, and hazard perception in driving", Accident Analysis and Prevention, 42, pp. 1240-1249.

Braithwaite, J.J., Watson, D.G., Jones, R., Rowe, M. (2013) "A guide for analysing electrodermal activity (EDA) \& skin conductance responses (SCRs) for psychological experiments", Psychophysiology 49, 1017-1034.

Cacioppo, J.T., Tassinary, L.G., Berntson, G. (2007) "Handbook of Psychophysiology", Cambridge University Press.

Cain, B. (2007) "A review of the mental workload literature (Report RTO-TR-HFM121-Part-II)", Toronto, Canada: Defence Research and Development.

Chen, Y., Persaud, B., Sacchi, E., Bassani, M. (2013) "Investigation of models for relating roundabout safety to predicted speed", Accident Analysis and Prevention, 50, pp. 196-203.

Cheng, A.S.K., Ng, T.C.K., Lee, H.C. (2011) "A comparison of the hazard perception ability of accident-involved and accident-free motorcycle riders", Accident Analysis and Prevention, 43, pp. 1464-1471.

Easa, S.M., Mehmood, A. (2006) "Optimizing geometric design of roundabouts: multiobjective analysis", Canadian Journal of Civil Engineering, 33 (1), pp. 29-40.

Daniels, S., Brijs, T., Nuyts, E., Wets, G. (2010) "Externality of risk and crash severity at roundabouts", Accident Analysis Prevention 42, 1966-1973.

De Winter, J. C. F., Happee, R., Martens, M. H., \& Stanton, N. A. (2014) "Effects of adaptive cruise control and highly automated driving on workload and situation awareness: A review of the empirical evidence", Transportation Research Part F: Traffic Psychology and Behaviour, 27, 196-217.

Dijksterhuis, C., Brookhuis, K. A., \& De Waard, D. (2011) "Effects of steering demand on lane keeping behaviour, self-reports, and physiology. A simulator study", Accident Analysis and Prevention, 43, 1074-1081.

Distefano, N., Leonardi, S., (2018) "A list of accident scenarios for three legs skewed intersections", IATSS Research. Vol. 42. Issue 3. 97-104. DOI: 10.1016/j.iatssr.2017.07.003.

Distefano, N., Leonardi, S., (2019) "Evaluation of the benefits of traffic calming on vehicle speed reduction", Civil Engineering and Architecture, 7 (4), 200-214. DOI: 10.13189/cea.2019.070403.

Distefano, N., Leonardi, S., Pulvirenti, G. (2018) "Factors with the greatest influence on drivers' judgment of roundabouts safety. An analysis based on web survey in Italy", IATSS Research, DOI: 10.1016/j.iatssr.2018.04.002. 
Distefano, N., Leonardi, S., Consoli, F. (2019a). "Drivers' Preferences for Road Roundabouts: A Study based on Stated Preference Survey in Italy", KSCE Journal of Civil Engineering, Vol. 23 (11), no. 2016, pp. 1-11. DOI: 10.1007/s12205-0191363-9.

Distefano, N., Leonardi, S., Pulvirenti, G. (2019b) "Italians' public opinion on road roundabouts: A web-based survey", Proceedings of the AIIT International Congress on Transport Infrastructure and Systems in a changing world (TIS), Roma.

Distefano, N., Leonardi, S., Pulvirenti, G. (2019c) "Home-school Travel: Analysis of Factors Affecting Italian Parents' Mode Choice", Civil Engineering and Architecture, 7 (3), 75-87. DOI: 10.13189/cea.2019.070302.

Elvik, R. (2003) "Effects on Road Safety of Converting Intersections to Roundabouts Review of Evidence from Non-U.S. Studies", Transportation Research Record, 1847, pp. 1-10.

Fernandes, P., Teixeira, J., Guarnaccia, C., Bandeira, J.M., Macedo, E., Coelho, M.C. (2018) "The potential of metering roundabouts: Influence in transportation externalities", Transportation Research Record, Vol. 2672 (25), pp. 21-34.

Fowles, D.C., Christie, M.J., Edelberg, R., Grings, W.W., Lykken, D.T., Venables, P.H. (1981) "Publication recommendations for electrodermal measurements", Psychophysiology 18(3):232-9.

Garbarino, M., Lai, M., Tognetti, S., Picard, R. W., \& Bender, D. (2014) "Empatica E3 - a wearable wireless multi-sensor device for real-time computerized biofeedback and data acquisition" International conference on wireless mobile communication and healthcare (pp. 39-42). Athens, Greece: IEEE. https://doi.org/10.4108/icst.mobihealth.2014.257418.

Gössling, S., Humpe, A., Litman, T., Metzler, D. (2019) "Effects of perceived traffic risks, noise, and exhaust smells on bicyclist behaviour: An economic evaluation", Sustainability (Switzerland), Vol. 11 (2), Art. N. 408.

Grayson, G., Maycock, G. (1988) "From proneness to liability", In J. A. Rothengatter \& R. A. de Bruin (Eds.), Road user behaviour, pp. 234-241. Assen, Netherlands: Van Gorcum.

Gross, F., Lyon, C., Persaud, B., Srinivasan, R. (2013) "Safety effectiveness of converting signalized intersections to roundabouts", Accident Analysis and Prevention, 50, pp. 234-241.

Horswill, M.S., McKenna, F.P. (2004) "Drivers' hazard perception ability: situation awareness on the road", In S. Banbury, S., Tremblay, S. (Eds.), A Cognitive Approach to Situation Awareness: Theory and Application, pp. 155-175.

Hu, W., McCartt, A.T., Jermakian, J.S., Mandavilli, S., 2014. Public Opinion, Traffic Performance, the Environment, and Safety after Construction of Double-Lane Roundabouts. Transportation Research Record: Journal of the Transportation Research Board, 2402, 47-55.

Leonardi, S., Distefano, N., Pulvirenti, G. (2019), "Multiple Correspondence Analysis (MCA) for the evaluation of risk perception of roundabouts for young people", European Transport \Trasporti Europei, Issue 72, Paper n 4, ISSN 1825-3997.

Ma, W., Liu, Y., Head, L., Yang, X. (2013) "Integrated optimization of lane markings and timings for signalized roundabouts", Transportation Research Part C: Emerging Technologies, 36, pp. 307-323.

McKenna, F. P., Crick, J. L. (1997) "Developments in hazard perception” (Report No. TRL297), Berkshire, UK: Transport Research Laboratory. 
Mehler, B., Reimer, B., Coughlin, J. F., \& Dusek, J. A. (2009) "Impact of incremental increases in cognitive workload on physiological arousal and performance in young adult drivers", Transportation Research Record: Journal of the Transportation Research Board, 2138, 6-12.

Nicholson, L.M., Turner, L., Slater, S.J, Abuzayd, H., Chriqui, J.F., Chaloupka, F. (2014) "Developing a measure of traffic calming associated with elementary school students' active transport", Transportation Research Part D: Transport and Environment, Vol. 33, pp. 17-25.

Persaud, B.N., Retting, R.A., Garder, P.E., Lord, D. (2001) "Safety effect of roundabout conversions in the United States: Empirical Bayes observational before-after study", Transportation Research Record Issue, 1751, pp. 1-8.

Poh, M.Z., Swenson, N.C., Picard, R.W. (2010) "A wearable sensor for unobtrusive, longterm assessment of electrodermal activity", IEEE Trans. Biomed. Eng. 57, 12431252 .

Retting, R.A., Luttrell, G. Russell, E.R., 2002. Public Opinion and Traffic Flow Impacts of Newly Installed Modern Roundabouts in the United States. ITE Journal, 72.9, 3037.

Sabey, B. E., Taylor, H. (1980) "The known risks we run: The highway", Supplementary Report SR 567. Transport and Road Research Laboratory, Crowthorne.

Seyle, H. (1956) "The Stress of Life”, McGraw-Hill, New York.

Vasudevan, V., Patel, T. (2017) "Comparison of discomfort caused by speed humps on bicyclists and riders of motorized", Sustainable Cities and Society, Vol. 35, pp. 669676

Villarejo, M. V., Zapirain, B. G., and Zorrilla, A. M. (2012) “A stress sensor based on galvanic skin response (GSR) controlled by ZigBee”, Sensors (Switzerland), 12(5), 6075-6101.

Vujanić, M., Antić, B., Pešić, D., Savićević, M. (2016) "Safety effectiveness of converting conventional intersections to roundabouts: Case study in the city of niŠ", Promet - Traffic \& Transportation, 28 (5), pp. 529-537.

Wickens, C. D., Lee, J. D., Liu, Y., \& Gordon-Becker, S. E. (2004) “An introduction to human factors engineering”, Upper Saddle River, NJ: Pearson Prentice Hall. 